\title{
Spectral domain optical coherence tomography documented rapid resolution of pseudophakic cystoid macular edema with topical difluprednate
}

This article was published in the following Dove Press journal:

Clinical Ophthalmology

23 January 2012

Number of times this article has been viewed

\section{KV Chalam \\ Vijay Khetpal \\ Chirag J Patel}

Department of Ophthalmology, University of Florida Jacksonville, FL, USA
Correspondence: KV Chalam Department of Ophthalmology, University of Florida Jacksonville, $580 \mathrm{~W}$ 8th Street, Tower 2, 3rd Floor, Jacksonville, FL 32209, USA

Tel + I 904244936 |

Fax + I 9042449391

Email kchalam@jax.ufl.edu
Introduction: Pseudophakic cystoid macular edema is a common cause of poor vision after cataract surgery, and topical corticosteroids and nonsteroidal anti-inflammatory drugs are used for its treatment. We investigated the effectiveness of difluprednate (Durezol ${ }^{\circledR}$, recently approved by the US Food and Drug Administration) in the treatment of cystoid macular edema, assisted with spectral domain optical coherence tomography (SD-OCT).

Case report: A 63-year-old African-American woman presented 6 weeks after uneventful cataract surgery in her left eye with decreased vision and associated distortion of the central visual field. Fluorescein angiogram and SD-OCT confirmed pseudophakic cystoid macular edema. Difluprednate was topically administered twice daily and monitored with serial imaging. Resolution was noted after 1 month of topical therapy, with improvement in visual acuity and resolution of distortion.

Conclusion: Difluprednate is an effective treatment for patients with severe pseudophakic cystoid macular edema. SD-OCT allows the physician to monitor resolution of the macular edema easily.

Keywords: cystoid macular edema, difluprednate, spectral domain optical coherence tomography, pseudophakic

\section{Introduction}

Cystoid macular edema (CME) is a common cause of visual loss after complicated cataract surgery, but may also occur in uncomplicated cases. ${ }^{1}$ Intraoperative vitreous loss, placement of an anterior chamber intraocular lens, and prolonged postoperative inflammation are some of the risk factors associated with $\mathrm{CME}{ }^{1}$ Patients experience diminished vision approximately 6-8 weeks after cataract surgery. Diagnosis is based on clinical findings, characteristic appearance on fundus fluorescein angiography, and optical coherence tomography. Common modalities of treatment currently include topical nonsteroidal anti-inflammatory drugs alone or in combination with topical corticosteroids for 3-4 months or until resolution of the macular edema. In patients with persistent CME, intravitreal injections of bevacizumab or triamcinolone have been used with success. ${ }^{1,2}$ In this case report, we describe rapid resolution of pseudophakic CME documented with spectral domain optical coherence tomography (SD-OCT, Spectralis ${ }^{\circledR}$, Heidelberg Engineering Inc, Carlsbad, CA) after topical administration of difluprednate $0.05 \%$ (Durezol $^{\circledR}$, Sirion Therapeutics, Tampa, FL), a fluorinated corticosteroid with reportedly greater intraocular penetration. ${ }^{3-6}$ 


\section{Case presentation}

A 63-year-old African-American woman with a history of hypertension, asthma, gastroesophageal reflux disease, anemia, and hyperlipidemia, was referred for evaluation of poor vision. Her past ocular history was unremarkable. Her best corrected visual acuity was 20/30 in each eye, but on glare testing decreased to 20/40 in the right eye and 20/60 in the left eye. Near vision examination revealed $\mathrm{J} 1+$ in each eye and color vision was full. Amsler and visual fields were normal. Motility and pupillary examinations were normal. Intraocular pressures were $16 \mathrm{mmHg}$ in the right eye and $18 \mathrm{mmHg}$ in the left eye. Anterior segment examination was notable for a nuclear sclerotic cataract in both eyes (left greater than right). Dilated fundus examination revealed minimal diabetic retinopathy with microaneurysms in the posterior pole.

Uneventful phacoemulsification was performed to her left eye and a B and L LI61 AO + 21.5 diopter lens was placed into the capsular bag. On postoperative day 1, the patient's visual acuity was 20/20 and intraocular pressure was $16 \mathrm{mmHg}$ in the left eye. A routine postoperative regimen of tobramycin and dexamethasone was started and tapered off over the next 4 weeks. Six weeks later, she presented with central visual field distortion and decreased vision in her left eye. Best corrected visual acuity in her left eye was $20 / 50+2$, with refraction of $-0.50+0.25 \times 005$. Slit lamp examination revealed a well centered intraocular lens without anterior chamber inflammation. Dilated fundus examination revealed diffuse macular edema.

SD-OCT revealed CME with intraretinal thickening and a central macular thickness of $819 \mu \mathrm{m}\left(0.64 \mathrm{~mm}^{3}\right)$ in her left eye (Figure 1A and C). Fundus fluorescein angiography confirmed severe CME in the late phase of the angiogram (Figure 1B).

Although pseudophakic CME may resolve spontaneously, topical difluprednate was initiated in the left eye twice daily given the severe cystic macular thickening. After 4 weeks of therapy, her best corrected visual acuity improved to 20/20 in the left eye and the CME resolved clinically. The intraocular pressure remained at $16 \mathrm{mmHg}$ in the left eye after 4 weeks of therapy. SD-OCT imaging showed complete resolution of CME with a central macular thickness of $310\left(0.24 \mathrm{~mm}^{3}\right.$, Figure 2A and C) Repeat fundus fluorescein angiography showed resolution of CME (Figure 2B).

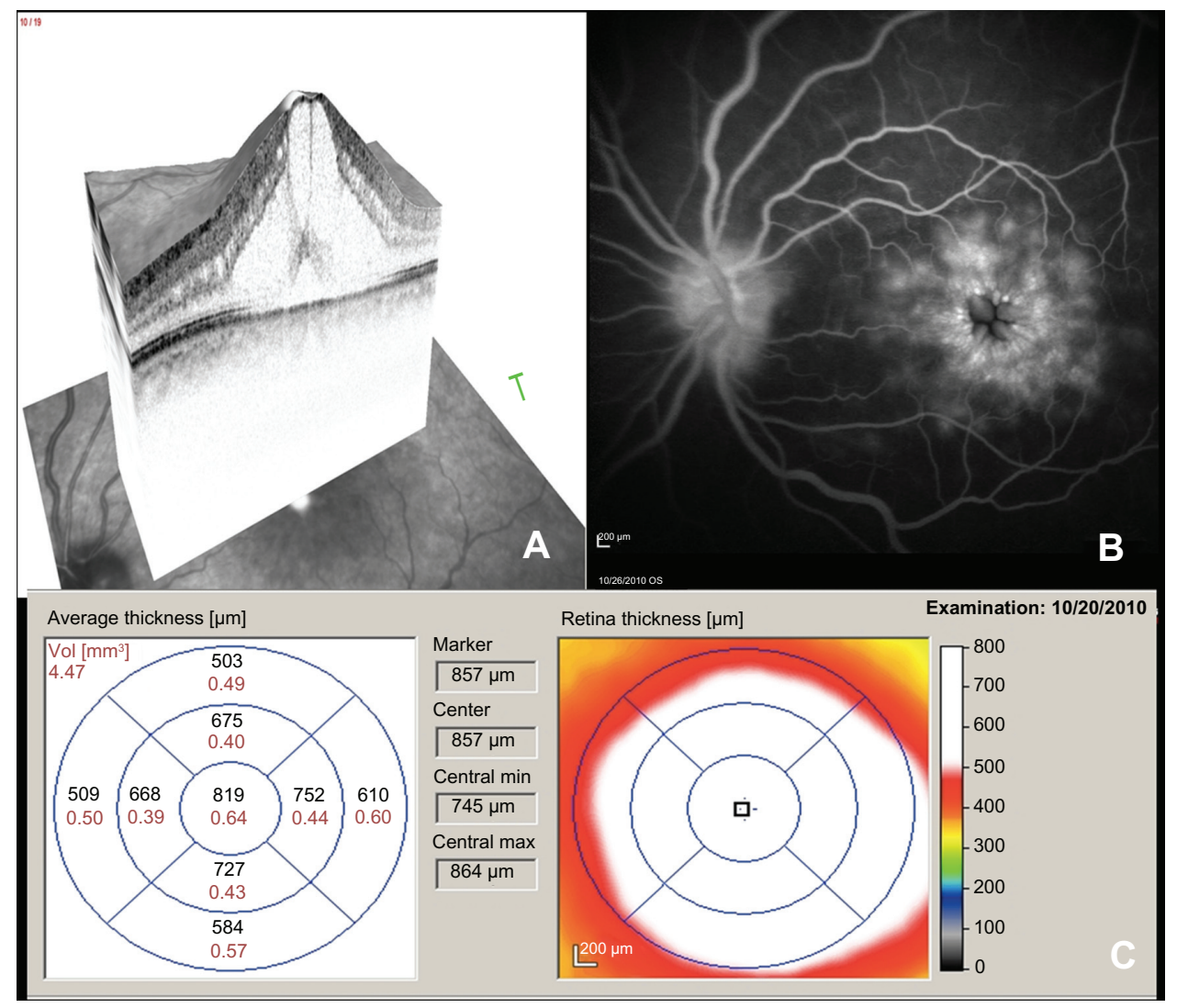

Figure I Spectral domain optical coherence tomography (A), fluorescein angiogram (B), and spectral domain optical coherence tomography thickness map (C) of the left eye prior to treatment with difluprednate. 


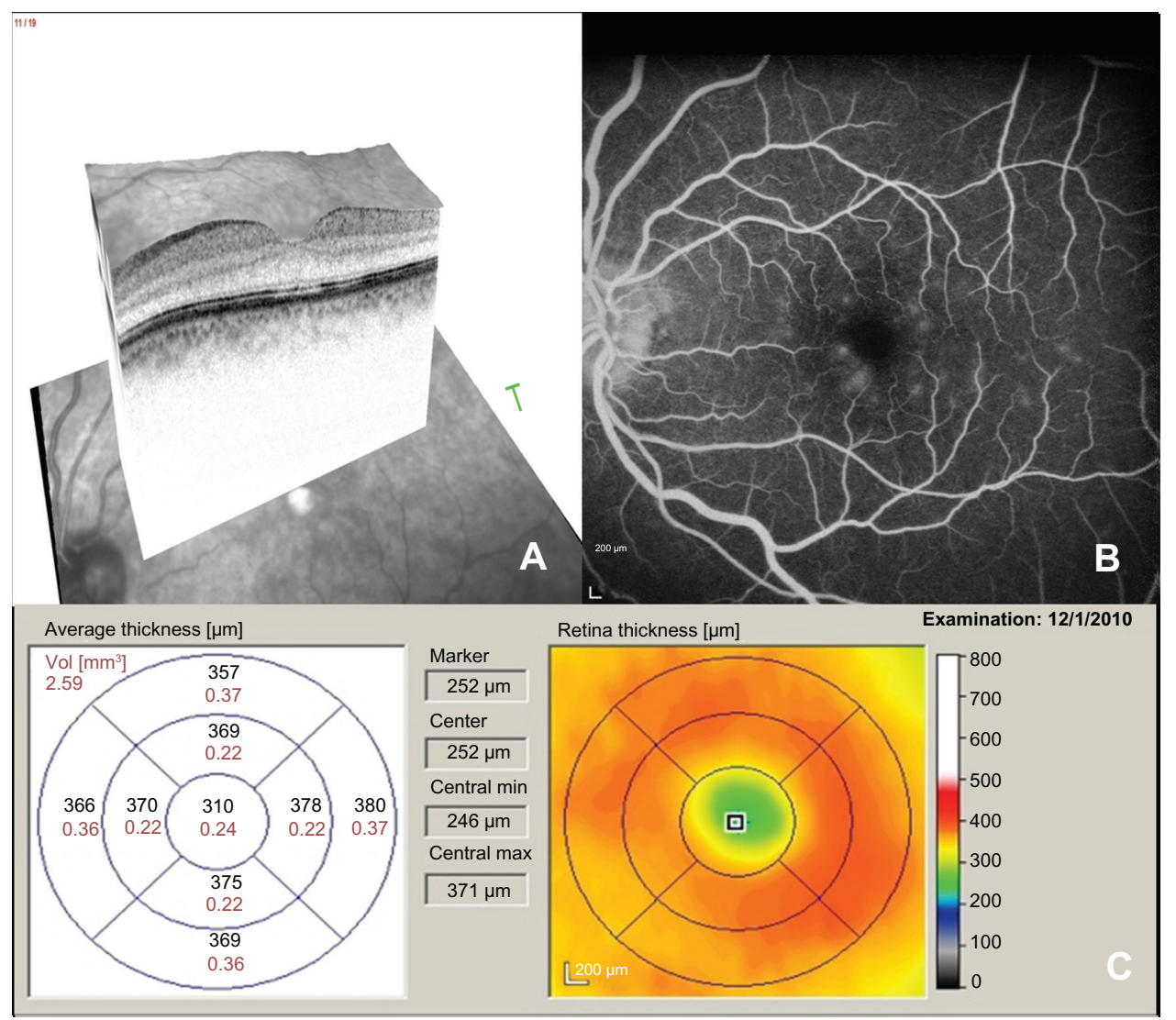

Figure 2 Spectral domain optical coherence tomography (A), fluorescein angiogram (B), and spectral domain optical coherence tomography thickness map (C) of the left eye after treatment with difluprednate.

\section{Discussion}

Pseudophakic CME is the most common cause of unexpected visual loss after cataract surgery. ${ }^{7,8}$ The incidence is estimated to range from $4 \%$ to $11 \%$ in the modern era. Because large randomized controlled trials with long-term follow-up are lacking, no standardized treatment or prophylactic regimen exists. ${ }^{7,8}$ Currently, topical nonsteroidal anti-inflammatory drugs and topical corticosteroids are first-line treatment modalities. Refractory cases of CME may be treated intravitreally with corticosteroids, nonsteroidal antiinflammatory drugs, or antivascular endothelial growth factor agents. ${ }^{2,7-10}$ We chose to administer topical difluprednate in view of its effectiveness, potency, and bioavailability. Furthermore, topical treatment modalities avoid the risks of intravitreal injections which include increased intraocular pressure and endophthalmitis.

Difluprednate (difluoroprednisolone butyrate acetate) is effective in the treatment of anterior uveitis, postoperative inflammation, and pain. However, its efficacy in postoperative CME is not described. Difluprednate, recently approved for inflammation and pain associated with ocular surgery in 2008, penetrates the corneal epithelium rapidly and effectively. ${ }^{3}$ Difluoroprednisolone butyrate, its active metabolite, has strong corticosteroid receptor agonist activity and is six times stronger than prednisolone in animal studies. ${ }^{3} \mathrm{It}$ is preserved with sorbic acid that is nontoxic to the corneal surface compared with benzalkonium chloride. ${ }^{3}$ Fluorination at the C-6 and C-9 positions increases glucocorticoid receptor binding affinity and the addition of butyrate and acetate esters at positions C-17 and C-21 improve corneal penetration compared with topical betamethasone., ${ }^{4,5}$ In addition, difluprednate is administered in an emulsion, thus increasing its bioavailability. ${ }^{4}$ The dose uniformity of difluprednate ophthalmic emulsion $0.05 \%$ was highly predictable compared with both branded and generic prednisolone (shaken or unshaken) acetate suspensions. ${ }^{6}$ Similar to other corticosteroids, difluprednate may cause a steroid-responsive intraocular pressure rise; however, its effect is minimal compared with other drugs in its class. ${ }^{10}$

We present a patient with severe CME with a central macular thickness of $819 \mu \mathrm{m}\left(0.64 \mathrm{~mm}^{3}\right)$ and the presence of subretinal fluid on OCT. Although pseudophakic CME may spontaneously resolve, severe cystic thickening warrants therapy. ${ }^{7,8}$ Our patient's visual acuity returned to normal after 
using difluprednate for 4 weeks and the ultrastructural changes in the fovea resolved with recovery of normal anatomy.

\section{Conclusion}

In summary, severe pseudophakic CME resolves rapidly after topical administration of difluprednate. SD-OCT was helpful both in identifying CME and monitoring of treatment with serial scans.

\section{Disclosure}

The authors report no conflicts of interest in this work.

\section{References}

1. Hariprasad SM, Akduman L, Clever JA, Ober M, Recchia FM, Mieler WF. Treatment of cystoid macular edema with the new-generation NSAID nepafenac 0.1\%. Clin Ophthalmol. 2009;3:147-154.

2. Chalam KV, Keshavamurthy R, Brar VS. Spectral domain OCT documented resolution of recalcitrant macular edema after intravitreal bevacizumab in branch retinal vein occlusion. Eur J Ophthalmol. 2008;18:831-833.

3. Korenfeld MS, Silverstein SM, Cooke DL, Vogel R, Crockett RS; Difluprednate Ophthalmic Emulsion 0.05\% (Durezol) Study Group. Difluprednate ophthalmic emulsion $0.05 \%$ for postoperative inflammation and pain. $J$ Cataract Refract Surg. 2009;35:26-34.
4. Foster CS, Davanzo R, Flynn TE, et al. Durezol (difluprednate ophthalmic emulsion $0.05 \%$ ) compared with Pred Forte $1 \%$ ophthalmic suspension in the treatment of endogenous anterior uveitis. $J$ Ocul Pharmacol Ther. 2010;2:475-483.

5. Tajika T, Waki M, Tsuzuki M, Kida T, Sakaki H. Pharmacokinetic features of difluprednate ophthalmic emulsion in rabbits as determined by glucocorticoid receptor-binding bioassay. J Ocul Pharmacol Ther. 2011;27:29-34.

6. Nakano S, Yamamoto T, Kirii E, Abe S, Yamashita H. Steroid eye drop treatment (difluprednate ophthalmic emulsion) is effective in reducing refractory diabetic macular edema. Graefes Arch Clin Exp Ophthalmol. 2010;248:805-810.

7. Shelsta HN, Jampol LM. Pharmacologic therapy of pseudophakic cystoid macular edema: 2010 update. Retina. 2011;31:4-12.

8. Flach AJ. The incidence, pathogenesis and treatment of cystoid macular edema following cataract surgery. Trans Am Ophthalmol Soc. 1998;96:557-634.

9. Spitzer MS, Ziemssen F, Yoeruek E, et al. Efficacy of intravitreal bevacizumab in treating postoperative pseudophakic cystoid macular edema. J Cataract Refract Surg. 2008;34:70-75.

10. Ahmadabadi HF, Mohammadi M, Beheshtnejad H, Mirshahi A. Effect of intravitreal triamcinolone acetonide injection on central macular thickness in diabetic patients having phacoemulsification. $J$ Cataract Refract Surg. 2010;36:917-922.
Clinical Ophthalmology

\section{Publish your work in this journal}

Clinical Ophthalmology is an international, peer-reviewed journal covering all subspecialties within ophthalmology. Key topics include: Optometry; Visual science; Pharmacology and drug therapy in eye diseases; Basic Sciences; Primary and Secondary eye care; Patient Safety and Quality of Care Improvements. This journal is indexed on

Submit your manuscript here: http://www.dovepress.com/clinical-ophthalmology-journal

\section{Dovepress}

PubMed Central and CAS, and is the official journal of The Society of Clinical Ophthalmology (SCO). The manuscript management system is completely online and includes a very quick and fair peer-review system, which is all easy to use. Visit http://www.dovepress.com/ testimonials.php to read real quotes from published authors. 